\title{
Estratégias lúdico-interativas para a promoção de hábitos alimentares saudáveis e práticas desportivas de escolares
}

Ludic-interactive strategies for promoting healthy eating and sporting habits of schoolchildren

\section{RESUMO}

$\mathrm{O}$ aumento do sobrepeso e obesidade em crianças e adolescentes são fatores de grande preocupação global. As escolas são ambientes favoráveis para prevenção do excesso de peso pelo estímulo ao consumo de alimentos saudáveis e à prática de atividade física. Esse trabalho objetivou verificar o estado nutricional de alunos da rede pública de João Pessoa, PB, Brasil, e divulgar os benefícios do exercício físico e da alimentação saudável por meio de atividades lúdicas e da discussão de temas relacionados às pirâmides alimentar e de atividade física. A análise do estado nutricional demonstrou que a maioria dos escolares avaliados apresentou estatura/idade (95,3\%) e peso/idade ou índice de massa corporal/idade adequados (70,9\%). Entretanto, aproximadamente $25 \%$ dos escolares apresentaram excesso de peso. Foi também observado que eles não conheciam os aspectos de proporcionalidade no consumo dos alimentos e nem a necessidade de diversificação dos exercícios físicos, embora os praticassem rotineiramente. As atividades lúdicas com a apresentação das pirâmides alimentar e de exercícios físicos, desenvolvidas durante o projeto de extensão, ressaltaram os benefícios da alimentação equilibrada associada à pratica regular e diversificada de exercícios físicos, necessários à manutenção de uma vida saudável e à prevenção de doenças.

Palavras-chave: Escolares. Obesidade. Alimentação saudável. Atividade física. Ações lúdicas.

\section{ABSTRACT}

The increased incidence of overweight and obesity in children and adolescents is a matter of concern worldwide. Schools are a favorable environment to help preventing overweight by encouraging the consumption of healthy food and the practice of physical activity. This extension study aimed to evaluate the nutritional status of students from a public school of João Pessoa, PB, Brazil, and disseminate the
Francisco Antônio de Oliveira Júnior

Doutorando em Ciências Fisiológicas na Universidade Federal da Paraíba, Brasil; técnico de laboratório do Departamento de Fisiologia e Patologia da Universidade Federal da Paraíba, Brasil. (junior.ltf@ gmail.com).

Vinícius José Baccin Martins

Doutor em Ciências Endocrinológicas pela Universidade Federal de São Paulo; professor adjunto do Departamento de Fisiologia e Patologia da Universidade Federal da Paraíba, Brasil. (vifisio@ yahoo.com.br).

Rita de Cássia da Silveira e Sá

Pós-doutora em Métodos Alternativos em Experimentação Animal pela Johns Hopkins Bloomberg School of Public Health, Baltimore, MD, USA; professora associada do Departamento de Fisiologia e Patologia da Universidade Federal da Paraíba, Brasil. (ritacassia.sa@bol. com.br).

Maria Regina de Freitas

Doutora em Farmacologia Celular e Molecular pela Université Louis Pasteur Strasbourg, França; professora associada do Departamento de Fisiologia e Patologia da Universidade Federal da Paraíba, Brasil. (rfreitas.ufpb@gmail. com). 
benefits of physical exercise and healthy eating through ludic activities and the discussion of topics related to the food pyramid and the pyramid of physical activity. The analysis of the nutritional status showed that the majority of schoolchildren presented adequate stature/ age $(95,3 \%)$ and weight/age or corporal mass index/age (70,9\%). However, approximately $25 \%$ of the subjects were overweight. It was also observed that they were unaware of proportionality of food intake and the necessity of diversification of physical exercises, despite practicing them routinely. The ludic activities involving the presentation of the food pyramid and the physical activity pyramid reinforced the benefits of healthy eating associated with regular practice of physical activities as a way to maintain a healthy life and prevent diseases.

Keywords: Schoolchildren. Obesity. Healthy eating. Physical activity. Ludic actions.

\section{INTRODUÇÃO}

A prática regular de atividade física e de uma alimentação saudável são fatores primordiais na prevenção de doenças crônicas, como as doenças cardíacas e o câncer, que figuram dentre as principais causas de morte entre os adultos (LICHTENSTEIN et al., 2006; CDCP, 2011). Vários estudos indicam que a inatividade física aliada a uma dieta rica em carboidratos e gorduras e pobre em vitaminas podem aumentar o risco à saúde, levando ao aparecimento de doenças crônicas, incluindo a pressão alta, o diabetes do tipo 2 e a obesidade (CDCP, 2011). Segundo a Organização Mundial da Saúde - OMS (WHO, 2016), a obesidade é uma doença caracterizada pelo acúmulo excessivo de gordura corporal com potencial prejuízo à saúde decorrente de fatores genéticos ou ambientais, como padróes dietéticos e de atividade física, ou fatores individuais de susceptibilidade biológica, que interagem na etiologia da patologia.

Os efeitos da obesidade nas crianças podem ter consequências prejudiciais à saúde, tanto durante a infância e adolescência, quanto na vida adulta, uma vez que promove o desenvolvimento precoce de doenças crônicas, tais com diabetes, hipertensão e dislipidemias (ENES; SLATER, 2010; LEE, 2009). Por isso, o sedentarismo e os 
hábitos alimentares de crianças e adolescentes são de grande interesse para a saúde pública (MALTA et al., 2009). Há um entendimento claro de que a atividade física na infância e adolescência reduz o risco de obesidade na própria infância e na fase adulta, o que enfatiza a relevância de atitudes que diminuam o sedentarismo e estimulem a prática saudável de esportes ou outras atividades físicas (GUTHOLD et al., 2010; MUST; TYBOR, 2005).

O aumento da prevalência de sobrepeso ou obesidade em crianças, jovens e adultos caracteriza essa doença crônica como uma epidemia global e um importante problema de saúde pública (WHO, 2016). Em 2014, 39\% da população mundial, ou seja, mais de 1,9 bilhões de adultos acima dos 18 anos de idade apresentaram sobrepeso e 600 milhóes, obesidade (13\%). Neste mesmo ano, 41 milhóes de crianças, abaixo de 5 anos de idade, apresentaram sobrepeso ou obesidade (WHO, 2014).

Dados divulgados nos Estados Unidos revelaram que a prevalência do sobrepeso em crianças de 6 a 11 anos de idade dobrou e em adolescentes, entre 12 a 19 anos, triplicou nas últimas duas décadas (HA et al., 2005; OGDEN et al., 2014). Na América Latina, entre 42,5 a 51,8 milhóes de crianças e adolescentes (20-25\% da população), com idade de 0-19 anos, estavam com sobrepeso ou obesidade (RIVERA et al., 2015). No Brasil, essa realidade não é muito diferente, pois estudos evidenciaram o aumento crescente do número de crianças e adolescentes com obesidade (ALVARENGA et al., 2013; JARDIM et al., 2017). Nos escolares, em particular, a obesidade se tornou um problema de graves consequências, já que o excesso de peso dificulta o processo de crescimento físico e aprendizagem motora do indivíduo, além de afetar a autoestima, que pode levar a um menor rendimento escolar (ARAÚJO, 2001; HUNT, 2008).

O hábito alimentar e a realização de atividade física por crianças e adolescentes são influenciados por fatores sociais distintos, envolvendo familiares, escolas, creches, comunidades, agências governamentais, imprensa, indústrias de bebidas e alimentícia, e a indústria do entretenimento virtual, cada qual com a sua contribuição específica no contexto comportamental dos jovens e crianças em relaçáo à alimentação e ao exercício físico (CDCP, 2011). O enfoque do papel das escolas dentro dessa realidade mostra que elas exercem uma 
função fundamental na busca por uma melhor qualidade de vida desses indivíduos, porque podem estabelecer um ambiente seguro e de apoio por meio de políticas promotoras de um comportamento saudável e que propiciem oportunidades de aprendizado e práticas de atividade física e de uma alimentação saudável (CDCP, 2011). Em relação à prática do exercício físico, a escola assume uma posição crítica de conscientização e realização de tal prática, visto que, às vezes, torna-se o único ambiente disponível para o engajamento dos escolares em algum tipo de esporte (ALVES, 2007; MAYER; WEVER, 2013). Essas açóes poderiam contribuir, por exemplo, para alterar o estilo de vida sedentário adotado por jovens e crianças, que preferem passar longas horas assistindo televisão ou utilizando o computador, ao invés de se engajarem em atividades saudáveis (ANDERSEN et al., 1998; ZANG et al., 2015).

Portanto, a manutençáo de um estilo de vida adequado desde a infância é um fator preponderante para o controle da obesidade. Muitos comportamentos exibidos na fase adulta são adquiridos e/ou consolidados nessa fase, o que torna necessário fazer uma avaliaçáo consciente dos elementos que compóem esses comportamentos. Além disso, é também relevante promover a incorporaçáo de um estilo de vida saudável de maneira gradual e duradoura, com ênfase na formação de hábitos alimentares adequados, e no estímulo à prática de atividades físicas (COELHO et al., 2012; KAIN et al., 2001; LAMAS; LORENZO, 2003; MELLO et al., 2004).

Devido à dificuldade de se obter sucesso no combate à obesidade em adultos, torna-se imprescindível investir na implementação de medidas preventivas e tratamento desta doença ainda na infância (MALTA; MORAIS NETO; SILVA JÚNIOR, 2011). Por esse motivo, os objetivos do presente estudo foram avaliar o estado nutricional de escolares de 10 a 13 anos de idade de uma escola pública de João Pessoa, Paraíba, e apresentar e discutir, de maneira lúdica e interativa, os benefícios da alimentação saudável e da prática cotidiana de exercícios físicos. 


\section{METODOLOGIA}

\section{Participantes}

O desenvolvimento das atividades propostas contou com a participaçáo de 86 escolares, de ambos os sexos (meninas $n=44$, meninos $n=42$ ), de 10 a 13 anos de idade, matriculados no $5^{\circ}$ ano de uma escola de tempo integral da rede municipal de ensino de Joáo Pessoa, Brasil. Todas as atividades foram realizadas com o consentimento da escola, na quadra de esportes do estabelecimento. $\mathrm{O}$ projeto do presente estudo foi submetido e aprovado pelo Comitê de Ética em Pesquisa da Universidade Federal da Paraíba (UFPB), sob parecer no 86162.

A execução das atividades foi coordenada por docentes e discentes extensionistas dos cursos de graduação em Nutrição e em Licenciatura em Educaçáo Física da UFPB.

\section{Análise antropométrica e avaliaçáo de hábitos alimentares e de atividades físicas}

Dados referentes ao peso, estatura e idade foram coletados para o cálculo do Índice de Massa Corporal (IMC) e determinação do estado nutricional dos escolares. De acordo com a OMS, o estado nutricional de crianças e adolescentes é considerado adequado quando altura está entre os valores de - 2 e 2 escore z para idade e sexo. Quando estatura/ idade e/ou IMC está abaixo de -2 é considerado subnutrição, e quando o peso/idade e/ou IMC está acima de 1 ou 2 escore z considera-se sobrepeso ou obesidade, respectivamente (MCDONALD et al., 2013; ONIS et al., 2006; WHO, 2006).

Escolares ( $\mathrm{n}=86$ ), de ambos os sexos, trajando o uniforme escolar, foram pesados em balança plataforma com capacidade para $180 \mathrm{~kg}$ e precisão de $100 \mathrm{~g}$ (modelo W-127 marca WISO). Durante o processo de pesagem, os participantes foram orientados a se posicionarem de maneira que o peso ficasse distribuído igualmente nos membros inferiores (LOHMAN, 1981). A estatura foi mensurada com o auxílio de um estadiômetro (modelo Séries-12 marca WISO) fixado na parede. Os dados antropométricos foram analisados utlizando-se o programa AntroPlus v1.0.2. Foram empregados os indicadores do estado nutricional estatura/idade e índice de massa corporal para idade 
(IMC/idade), adotando-se a curva de 2006 da OMS como referência (WHO, 2006).

Informações sobre hábitos e preferências alimentares e de atividades físicas dos escolares $(n=82)$ foram obtidas por meio da aplicação de um questionário simplificado de 18 perguntas, respondido por meio de respostas do tipo SIM ou NÃO. Um Questionário Alimentar do Dia Anterior (QUADA), baseado em Assis et al. (2009), também foi aplicado, ressaltando alguns alimentos consumidos pelos escolares $(\mathrm{n}=47)$ por refeição diária. Responderam aos questionários e tiveram as medidas antropométricas aferidas, os escolares que apresentaram o Termo de Consentimento Livre e Esclarecido (TCLE), devidamente assinado por seu responsável legal. Nos dias de aplicação, alguns escolares não compareceram à escola, por motivos não relacionados ao estudo e, por isso, não responderam aos questionários.

As estratégias utilizadas na execução das atividades propostas priorizaram a integração e participação dos escolares na construçáo de um processo educacional interativo e dinâmico, visando, como ferramenta de trabalho, o emprego de atividades lúdicas baseadas em jogos que despertassem o interesse sobre os temas abordados.

\section{Atividade lúdica e a pirâmide alimentar}

Uma atividade lúdica interativa foi realizada com os escolares perfilados em duas equipes, na qual cada time tinha o objetivo de montar um cardápio de acordo com suas preferências alimentares. Para a construção do cardápio, foram preparadas imagens, coladas em cartolina, de vários tipos de alimentos constantes da pirâmide alimentar, a saber: verduras, frutas, óleos vegetais, carnes, peixes, ovos, doces, biscoitos recheados, pirulitos e batatas fritas, entre outros.

A atividade proposta foi desenvolvida da seguinte maneira: ao ouvir uma música (relacionada com o tema alimentação saudável), o primeiro jogador de cada fila, que segurava uma bola na mão, foi instruído a passá-la, por entre as pernas, ao jogador imediatamente atrás dele, e assim sucessivamente, até chegar ao último participante, que entáo correu com a bola para o início da fila, reiniciando a brincadeira. No decorrer da atividade, a música ia sendo interrompida, aleatoriamente, e o jogador que estivesse com a bola na mão (por 
ocasião da interrupção), dirigia-se à mesa que continha as bandejas com as figuras de alimentos. Em seguida, o participante escolhia uma das figuras disponíveis, que representasse o alimento mais consumido ou preferido por ele no seu dia-a-dia, e a colocava no prato para compor o cardápio de sua equipe.

Depois da participação de todos na composição dos pratos, os escolares foram divididos em grupos de 5 a 7 participantes, por assistente, para o início da segunda etapa da atividade. Esta envolveu a explicação da nova pirâmide alimentar (WILLETT; STAMPFER, 2003), adaptada para crianças e adolescentes, com o objetivo de apresentar e discutir as recomendaçóes nutricionais estabelecidas pela OMS (WHO, 2004), e voltadas à realidade da população local (BRASIL, 2014). Após o encerramento da discussão, foi realizada uma simulação da pirâmide alimentar, na qual os alimentos escolhidos pelos estudantes foram fixados de acordo com o nível correspondente já preconizado/recomendado. Foi estabelecida uma pontuação aleatória para os diferentes níveis da mesma, em uma escala decrescente, sendo os alimentos encontrados na base da pirâmide e que devem ser consumidos com maior frequência, com maior pontuação, isto é, seis pontos para cereais, páes integrais e azeite, cinco pontos para hortaliças e frutas, quatro pontos para legumes, três pontos para peixes, aves e ovos, dois pontos para leite e produtos lácteos, e um ponto para gorduras saturadas, carne vermelha, açúcares e doces. Assim, os alimentos do topo da pirâmide, que devem ser evitados, ficaram com a menor pontuação, ou seja, apenas um ponto. A equipe vencedora foi aquela com maior pontuação, obtida pela soma dos pontos correspondentes aos alimentos selecionados.

Todos os escolares receberam como premiação simbólica blocos de anotaçóes com o logotipo do projeto e caneta. Com isso, eles foram estimulados a fazer perguntas relacionadas ao tema desenvolvido com o objetivo de reforçar o aprendizado adquirido durante as atividades. As perguntas foram depositadas em uma urna e respondidas posteriormente.

\section{Atividade lúdica e a pirâmide da atividade física}

A pirâmide da atividade física para crianças e adolescentes, adaptada de Martins (2009), foi utilizada como instrumento para discussão sobre 
os benefícios do exercício físico, seguido da realização de atividades que visaram a contextualizaçáo do tema abordado.

Inicialmente os escolares foram divididos em grupos de 5 a 7 participantes por assistente para explanação da pirâmide da atividade física para crianças e adolescentes. Em seguida, foram reagrupados e subdivididos em dois grandes grupos para responderem a perguntas, sorteadas aleatoriamente de um banco de questóes, direcionadas à exploração do conteúdo abordado. Para desenvolver essa atividade, um representante de cada grupo foi orientado a disputar uma corrida, cujo o vencedor conquistaria o direito de responder à pergunta e, consequentemente, ganhar a pontuação correspondente. Após cada resposta, o conteúdo das perguntas ia sendo analisado e discutido com os participantes.

Em sequência ao jogo de perguntas e respostas, foi realizado um circuito misto de atividades, compreendendo as seguintes etapas: 1) pular argolas; 2) saltar barreiras; 3) passar por um túnel; 4) caminhar equilibrando um ovo numa colher; 5) atravessar uma trave de equilíbrio; 6) correr cerca de $200 \mathrm{~m}$; 7) fazer 30 polichinelos; 8) realizar uma acrobacia de elasticidade denominada "ponte" (Figura 1). De acordo com as regras estabelecidas, ao encerrar uma determinada etapa do circuito, o participante deveria tocar na máo do colega à sua frente para que o mesmo pudesse realizar a etapa seguinte, e assim sucessivamente, até o término de todas as atividades. A equipe vencedora foi aquela que mais rapidamente completou o circuito.

No último desafio, cada grupo escolheu representantes para disputa de força em um cabo de guerra, valendo o dobro da maior pontuaçáo obtida na fase anterior. Pela imposição de força conjunta de cada lado do cabo, venceu o grupo que conseguiu trazer os oponentes para o seu lado. Por fim, o somatório da pontuação em todas as fases determinou o grupo vencedor. Os escolares receberam como brinde simbólico adesivos com a mascote do projeto e a indicaçáo de $1^{\circ}$ e $2^{\circ}$ lugares. 
Figura 1 - Circuito misto de atividades físicas.

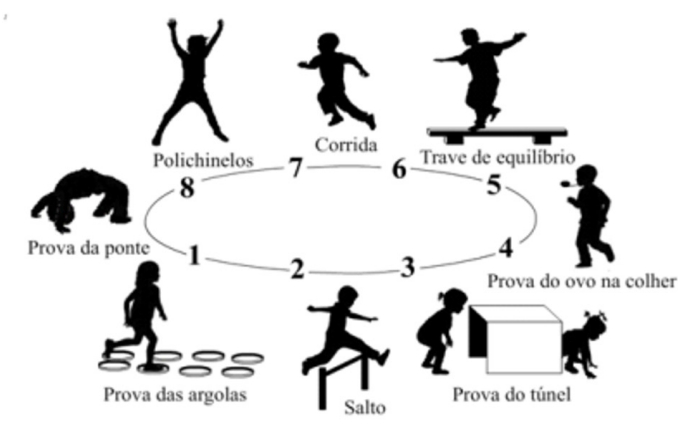

Fonte: Os autores (2016).

\section{Material didático}

Durante o período de coleta das variáveis antropométricas e realização das oficinas, foram distribuídos materiais didáticos, desenvolvidos pelo grupo de docentes e assistentes extensionistas discentes da UFPB. Estes materiais tinham uma abordagem lúdica e apropriada ao público alvo, priorizando a associação da alimentação saudável e da prática de atividade física, nas formas de folhetos de atividades com jogos e brincadeiras (Imagem 1) e folhetos instrucionais (Imagem 2).

No encerramento das atividades, foi distribuída uma cartilha com informaçóes e orientações sobre a prática de exercícios físicos e o consumo saudável de alimentos. 
Imagem 1 - Folheto sobre atividade física e alimentação saudável com jogos e brincadeiras.

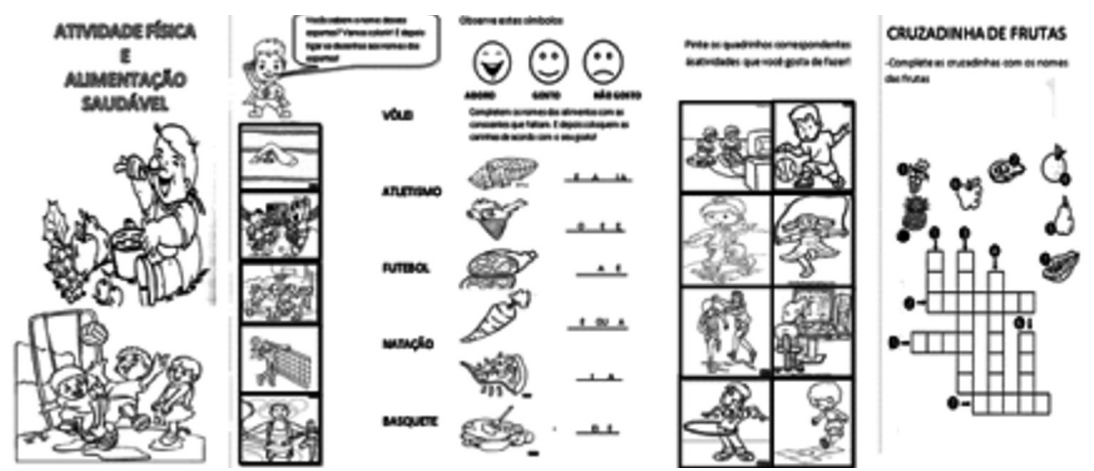

Fonte: Os autores (2016).

Imagem 2 - Folheto instrucional sobre alimentação saudável e atividade física.

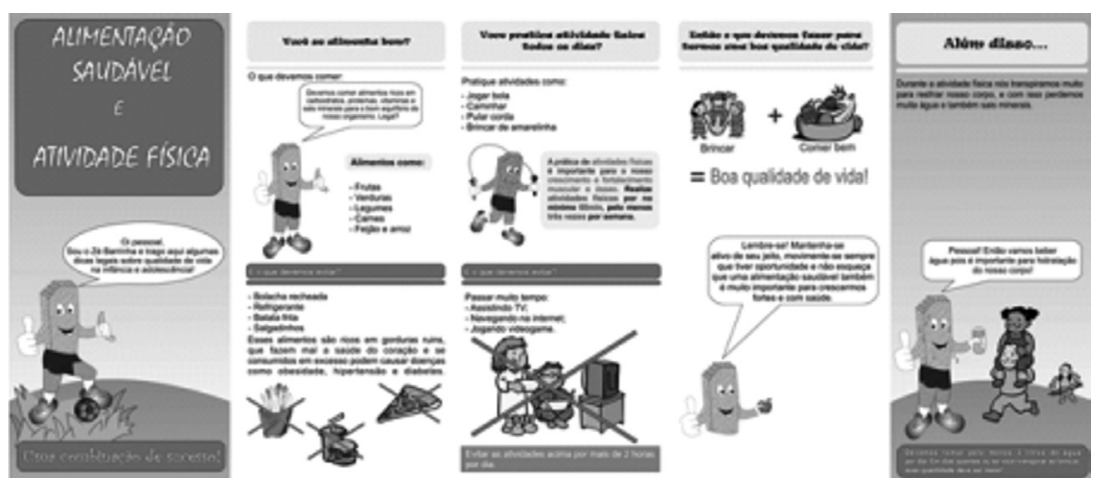

Fonte: Os autores (2016).

\section{Análise estatística}

Os dados antropométricos foram analisados por meio do teste $\mathrm{T}$ independente para as variáveis com distribuição normal e do teste do qui-quadrado para variáveis categóricas. As análises foram realizadas utilizando o software SPSS versão 20.0 (IBM). Foi considerado estatisticamente significante o valor de $\mathrm{p}<0,05$. 


\section{RESULTADOS}

A tabela 1 apresenta as características antropométricas dos alunos participantes do projeto. Não foram observadas diferenças significativas na média de idade, peso, altura e estatura/idade, IMC/ idade e IMC entre os sexos.

Tabela 1 - Variáveis antropométricas dos escolares, de 10 a 13 anos de idade, matriculados no quinto ano de uma escola da rede municipal da cidade de João Pessoa-PB, Brasil.

\begin{tabular}{llllll}
\hline & \multicolumn{3}{l}{ Meninas $(\mathrm{n}=44)$} & \multicolumn{2}{l}{ Meninos $(\mathrm{n}=42)$} \\
& Média & DP & Média & DP & P valor* \\
\hline Idade (anos) & 10,86 & 0,55 & 11,05 & 0,88 & 0,218 \\
Peso (kg) & 36,29 & 7,94 & 34,61 & 7,06 & 0,303 \\
Altura (cm) & 142,36 & 8,53 & 139,83 & 5,87 & 0,115 \\
Estatura/idade (z escore) & $-0,37$ & 1,15 & $-0,43$ & 0,93 & 0,778 \\
IMC/idade (z escore) & 0,03 & 1,11 & 0,05 & 1,43 & 0,936 \\
IMC (kg/m2) & 17,78 & 2,67 & 17,64 & 3,05 & 0,823 \\
\hline
\end{tabular}

Fonte: Os autores (2016).

*Teste $\mathrm{T}$ independente. $\mathrm{DP}=$ desvio padrão. $\mathrm{IMC}=$ índice de massa corporal.

A maioria das meninas avaliadas apresentou estatura/idade adequada $(90,9 \%)$, mas quatro delas $(9,1 \%)$ tiveram estatura/idade abaixo de $-2,0$ escore Z (Tabela 2). Nenhum menino obteve estatura/idade menor que -2,0 escore $Z$. A maioria das meninas (70,5\%) apresentou IMC/idade adequado, $25 \%$ excesso de peso e 4,5\%, déficit de peso. Os meninos exibiram prevalências similares às meninas (qui-quadrado $=0,018, \mathrm{p}=0,991$ ), sendo 71,4\% com IMC/idade adequado, 23,8\% com excesso de peso, e 4,8\%, com déficit de peso. 
Tabela 2 - Estado nutricional dos escolares, de 10 a 13 anos de idade, matriculados no quinto ano de uma escola da rede municipal da cidade de João Pessoa, PB, Brasil.

\begin{tabular}{lllll}
\hline & Meninas n(\%) & Meninos $\mathrm{n}(\%)$ & Total $\mathrm{n}(\%)$ & P valor \\
\hline $\begin{array}{l}\text { Estatura/idade } \\
\text { adequada }\end{array}$ & $40(90,9)$ & $42(100)$ & $82(95,3)$ & \\
$\begin{array}{l}\text { Déficit de estatura } \\
\text { Total }\end{array}$ & $4(9,1)$ & $0(0)$ & $4(4,7)$ & $0,117^{*}$ \\
$\begin{array}{l}\text { Peso/idade ou } \\
\text { IMC/idade adequados }\end{array}$ & $34(100)$ & $42(100)$ & $86(100)$ & \\
$\begin{array}{l}\text { Déficit de peso } \\
\text { Excesso de peso }\end{array}$ & $2(4,5)$ & $2(4,8)$ & $4(4,7)$ & $0,991^{* *}$ \\
Total & $11(25,0)$ & $10(23,8)$ & $21(24,4)$ & \\
\hline
\end{tabular}

Fonte: Os autores (2016).

*Teste exato de Fisher. ${ }^{* *}$ Qui-quadrado de Pearson. IMC= índice de massa corporal

A tabela 3 apresenta as perguntas feitas aos escolares sobre hábitos alimentares e atividade física. O questionário possui perguntas relacionáveis (contra-prova), ou seja, uma pergunta pode comprovar a informação contida na questão anterior, e revelou que $100 \%$ dos participantes $(n=82)$ gostam de frequentar as aulas de Educação Física. A maioria (83\%) pratica atividades físicas fora da escola, apesar de também estar envolvida em atividades sedentárias, como assistir televisão e utilizar o computador, por mais de 2 horas diárias (87\%), acompanhado do consumo de alimentos (87\%). Em relação aos questionamentos relacionados à alimentação, foram relatados hábitos saudáveis, como comer frutas todos os dias (78\%), retirar gordura excessiva de alimentos (51\%) e não consumir diariamente refrigerantes $(77 \%)$ ou frituras $(76 \%)$. Cerca de metade dos escolares (54\%) considera sua alimentação saudável, entretanto, grande parte (72\%) considera que precisa melhorar sua alimentação. Finalmente, cerca de $73 \%$ relatou já haver recebido algum tipo de orientação sobre alimentação saudável, e condizente com esta observação, a maioria relatou possuir conhecimentos sobre alimentos prejudiciais (82\%) e benéficos (77\%) à saúde do corpo. 
Tabela 3 - Questionamentos sobre atividade física e hábitos alimentares de escolares na faixa etária de 10 - 13 anos de idade $(\mathrm{n}=82)$

\begin{tabular}{|c|c|c|c|}
\hline & Perguntas & $\operatorname{Sim}(\%)$ & Não (\%) \\
\hline 01 & $\begin{array}{l}\text { Você gosta de frequentar as aulas de Educação } \\
\text { Física? }\end{array}$ & $82(100 \%)$ & $0(0 \%)$ \\
\hline 02 & $\begin{array}{l}\text { Você pratica atividades físicas fora da escola com } \\
\text { orientaçáo profissional (professor, instrutor de } \\
\text { academia)? }\end{array}$ & $34(41 \%)$ & $48(59 \%)$ \\
\hline 03 & $\begin{array}{l}\text { Você pratica atividade física fora da escola sem } \\
\text { orientação profissional (correr, fazer caminhada } \\
\text { etc.)? }\end{array}$ & $68(83 \%)$ & $14(17 \%)$ \\
\hline 04 & $\begin{array}{l}\text { Você costuma vir a pé ou de bicicleta para a } \\
\text { escola? }\end{array}$ & $64(78 \%)$ & $18(22 \%)$ \\
\hline 05 & Você costuma ajudar nos afazeres domésticos? & $59(72 \%)$ & $23(28 \%)$ \\
\hline 06 & $\begin{array}{l}\text { siste televisão ou fica no computador } \\
\text { s de duas horas diárias? }\end{array}$ & $71(87 \%)$ & $11(13 \%)$ \\
\hline 07 & $\begin{array}{l}\text { Você costuma se alimentar quando está } \\
\text { assistindo televisão ou utilizando o computador? }\end{array}$ & $71(87 \%)$ & $11(13 \%)$ \\
\hline 08 & Você toma refrigerante diariamente? & $19(23 \%)$ & $63(77 \%)$ \\
\hline 09 & Você come lanches com frituras diariamente? & $20(24 \%)$ & $62(76 \%)$ \\
\hline 10 & $\begin{array}{l}\text { Você costuma comer frutas e verduras } \\
\text { diariamente? }\end{array}$ & $64(78 \%)$ & $18(22 \%)$ \\
\hline 11 & $\begin{array}{l}\text { ma colocar mais sal nos alimentos } \\
\text { ervidos em seu prato? }\end{array}$ & $11(13 \%)$ & $71(87 \%)$ \\
\hline 12 & $\begin{array}{l}\text { Você costuma tirar a gordura aparente das } \\
\text { carnes ou a pele dos frangos antes de consumi- } \\
\text { los? }\end{array}$ & $42(51 \%)$ & $40(49 \%)$ \\
\hline 13 & $\begin{array}{l}\text { Você costuma fazer lanches entre as refeiçôes } \\
\text { (café/almoço/jantar)? }\end{array}$ & $69(84 \%)$ & $13(16 \%)$ \\
\hline 14 & Você considera sua alimentação saudável? & $44(54 \%)$ & $38(46 \%)$ \\
\hline 15 & $\begin{array}{l}\text { Você acredita que precisa melhorar a } \\
\text { alimentaçáo? }\end{array}$ & $59(72 \%)$ & $23(28 \%)$ \\
\hline 16 & $\begin{array}{l}\text { Você já recebeu algum tipo de orientaçáo sobre } \\
\text { alimentação saudável? }\end{array}$ & $60(73 \%)$ & $22(27 \%)$ \\
\hline 17 & $\begin{array}{l}\text { Você possui conhecimentos sobre alimentos que } \\
\text { sejam prejudiciais à saúde? }\end{array}$ & $67(82 \%)$ & $15(18 \%)$ \\
\hline 18 & $\begin{array}{l}\text { Você possui conhecimentos sobre alimentos que } \\
\text { sejam benéficos para o corpo? }\end{array}$ & $63(77 \%)$ & $19(23 \%)$ \\
\hline
\end{tabular}

Fonte: Os autores (2016). 
O consumo de alguns tipos de alimentos está evidenciado na Tabela 4. A avaliação dos dados do QUADA revelou o consumo de arroz, feijão, carne bovina ou frango e frutas - alimentos considerados essenciais (FISBERG et al., 2004) - no almoço e jantar pela maioria dos escolares. Quanto aos itens de alto teor calórico, foi observado que quase metade dos escolares consome regularmente biscoitos, guloseimas e refrigerantes.

Tabela 4 - Alimentos e bebidas consumidos pelos escolares $(n=47)$ em cada refeição.*

\begin{tabular}{lllllll}
\hline Alimentos/bebidas & Desjejum & Lanche & Almoço & Lanche & Jantar & Ceia \\
\hline Arroz & 0,0 & 5,9 & 100,0 & 2,9 & 70,6 & 8,8 \\
Feijăo & 0,0 & 2,9 & 88,2 & 0,0 & 50,0 & 5,9 \\
Carne/frango & 0,0 & 8,8 & 88,2 & 11,8 & 76,5 & 14,7 \\
Peixe & 0,0 & 5,9 & 50,0 & 0,0 & 38,2 & 5,9 \\
Ovo & 41,2 & 29,4 & 26,5 & 8,8 & 41,2 & 8,8 \\
Verduras & 5,9 & 8,8 & 38,2 & 0,0 & 23,5 & 2,9 \\
Frutas & 67,6 & 76,5 & 23,5 & 67,6 & 5,9 & 50,0 \\
Batata frita & 0,0 & 5,9 & 2,9 & 8,8 & 2,9 & 5,9 \\
Pizza & 2,9 & 8,8 & 2,9 & 14,7 & 29,4 & 29,4 \\
Páo e cereais & 76,5 & 41,2 & 0,0 & 38,2 & 29,4 & 26,5 \\
Leite & 47,0 & 23,5 & 0,0 & 29,4 & 26,5 & 23,5 \\
Achocolatado & 29,4 & 17,6 & 14,7 & 26,5 & 11,8 & 23,5 \\
Iogurte & 41,2 & 41,2 & 0,0 & 55,9 & 0,0 & 55,9 \\
Biscoitos/guloseimas & 8,8 & 14,7 & 0,0 & 47,1 & 5,9 & 41,2 \\
Suco & 11,8 & 20,6 & 41,2 & 35,3 & 32,3 & 17,6 \\
Refrigerante & 17,6 & 14,7 & 38,2 & 23,5 & 23,5 & 32,3 \\
\hline
\end{tabular}

Fonte: Os autores (2016).

*Os valores numéricos representam o percentual de escolares que consumiram o referido alimento em cada refeição.

\section{DISCUSSÃO}

$\mathrm{Na}$ fase escolar, o sobrepeso e a obesidade costumam levar crianças e adolescentes a um sentimento de baixa autoestima aliado a um 
comportamento de exclusão e de desmotivação nos estudos. Assim, a ideia de incentivá-los a realizarem algum tipo de atividade física e/ou esporte, fazendo com que isso se torne algo prazeroso e de inserção, além de despertar o interesse pelo consumo de alimentos saudáveis, sem a conotação de um regime de restriçóes e sacrifício, foi o objetivo central desse trabalho, visando a melhoria na qualidade de vida, saúde e educação, para além da sala de aula.

A antropometria é um importante método de avaliação do estado nutricional e de saúde, não invasivo, de baixo custo, que tem sido amplamente utilizado para monitorar o desenvolvimento de crianças (WHO, 1995). Segundo a OMS (ONIS et al., 2007), o crescimento e dimensôes corporais, em todas as idades, refletem o estado de saúde e bem-estar dos jovens e das populaçóes em geral. Portanto, a observaçáo de indicadores antropométricos fora dos valores recomendados sugere uma investigação clínica dos indivíduos afetados para identificar as possíveis causas das alteraçóes e a melhor forma de tratamento.

Apesar da maior parte dos escolares do presente estudo apresentar estatura e idade dentro da faixa normal para o sexo, a baixa estatura foi observada em cerca de $10 \%$ das meninas avaliadas. A baixa estatura pode ser consequência de vários fatores, incluindo o menor consumo energético-proteico e o aumento da prevalência de infecçóes. É também conhecido que a carga genética pode influenciar o crescimento em estatura, porém, os fatores ambientais exercem um papel muito mais importante na determinação da estatura final em comparação ao componente genético (AMIGO; BUSTOS, 1994). Além disso, a baixa escolaridade materna é outro fator que pode estar associado à baixa estatura, pois relaciona-se às condiçóes insalubres de moradia, conhecimentos básicos de higiene, falta de acesso médico e à menor renda (MARTINS et al., 2011; SAWAYA et al., 2003). Em uma revisão sistemática, Sousa e colaboradores (2016) demonstraram que a baixa estatura e o baixo peso corporal em crianças brasileiras acometem principalmente populaçóes marginalizadas com graves problemas sociais e de saúde pública. No presente estudo, foi evidenciado que aproximadamente $25 \%$ dos escolares apresentaram excesso de peso, de forma similar à média nacional, cuja prevalência em adolescentes de 10 a 19 anos é de 20,5\% para sobrepeso e 4,9\% para obesidade (IBGE, 2010). Esses dados podem ser parcialmente explicados pelos hábitos alimentares dos escolares, que apresentam grande consumo de 
bebidas ricas em açúcar e baixo consumo de verduras, aliada à prática de atividades sedentárias por mais de duas horas/dia.

A obesidade é causada por um desequilíbrio energético quando o excesso de calorias ingeridas excede o gasto energético, aumentando, assim, o peso corporal (WHO, 2016). Sabe-se que esse desequilíbrio tem origem multifatorial, como falta de exercício físico, consumo em excesso de alimentos com alta densidade energética, além de aspectos socioeconômicos e culturais (WHO, 2016). No passado, a obesidade era encontrada em populaçôes abastadas com disponibilidade abundante de energia, ao passo que hoje está associada a países em desenvolvimento, com baixa renda per capita e baixa qualidade alimentar (VELASQUES et al., 2011). Além disso, estudos recentes destacaram a coexistência entre subnutrição e obesidade em famílias com baixa renda (SOUSA et al., 2016). Nesses estudos, foram observados que membros da mesma família apresentaram obesidade ou baixa estatura, ou seja, mães com obesidade e os filhos com baixa estatura (BARQUERA et al., 2007), bem como sobrepeso ou obesidade concomitante à baixa estatura no mesmo indivíduo (FERNALD; NEUFELD, 2007). Várias modificaçôes nos sistemas fisiológicos, como aumento de cortisol e diminuição de hormônio tireoidiano contribuem para explicar parcialmente estes achados, que levam a uma adaptação voltada para economia de energia, que resulta em déficit de crescimento e acúmulo de gordura corporal (MARTINS et al., 2011).

Estudos realizados em escolas públicas e particulares revelaram resultados conflitantes em relaçáo à alimentaçáo e o IMC dos escolares. Por exemplo, em Santa Catarina, um programa de intervenção nutricional, desenvolvido com o objetivo de promover hábitos alimentares saudáveis em escolares do ensino médio, evidenciou a ausência de alteraçôes significativas do IMC, embora tenham ocorrido modificaçóes na conduta e nas práticas alimentares. Foi observado que, nas escolas particulares, houve diminuição do consumo de biscoitos recheados trazidos de casa e, na escola pública, ocorreu o aumento do consumo de merenda escolar e de frutas (GABRIEL et al., 2008). Por outro lado, outros estudos mostraram a ocorrência de taxas menores de sobrepeso e obesidade em escolas que adotaram programas de nutrição, promovendo hábitos alimentares mais saudáveis e maior frequência de atividade física em relação às escolas 
que náo desenvolveram tais programas (MAYER; WEBER, 2013; VEUGELERS; FITZGERALD, 2005). No presente estudo, a análise das informaçôes obtidas nos questionários indicou que os escolares estão tendo acesso a uma dieta razoavelmente adequada de alimentos, apesar de cerca de $25 \%$ terem apresentado sobrepeso e, $5 \%$, déficit de peso. Além disso, ficou evidenciado que os alunos possuem conhecimento sobre a importância do consumo de alimentos essenciais, como arroz, feijão, carnes e frutas, embora apresentem baixo consumo de verduras, além de consumirem com frequência alimentos de alto valor calórico e desprovidos de nutrientes, como biscoitos e guloseimas.

O aumento do consumo de alimentos ricos em açúcar e gordura, considerados de baixo valor nutricional (pobres em vitaminas e minerais), associado ao baixo consumo de frutas e verduras (alimentos de menor densidade energética), favorece a obesidade (TRICHES; GIUGLIANI, 2005) e pode ser associado ao desenvolvimento de doenças crônicas (COELHO et al., 2012; MENDONÇA; DOS ANJOS, 2004). Portanto, o envolvimento familiar, comunitário e escolar na educação alimentar das crianças é fundamental para que elas adquiram hábitos alimentares saudáveis, consumindo adequadamente frutas e verduras e menos alimentos calóricos para diminuir os riscos à saúde ao atingirem a idade adulta (HA et al., 2005).

A pirâmide alimentar é um instrumento de educação nutricional importante, utilizado na pesquisa de abordagem e avaliação de padrôes dietéticos e atributos de indivíduos e grupos (KENNEDY et al., 2001; TAVELLI et al., 1998). Ela trabalha com três conceitos básicos, relevantes para uma orientação alimentar consciente, a saber: variedade, proporcionalidade e moderaçấo (GOLDBERG et al., 2007). A discussão sobre alimentação saudável com base na pirâmide alimentar, em conjunto com a análise dos resultados obtidos na montagem da pirâmide construída pelos escolares, revelou que estes tinham conhecimento prévio sobre a pirâmide alimentar, sabiam distinguir os alimentos saudáveis dos não saudáveis, mas desconheciam os aspectos de proporcionalidade e moderação de consumo dos alimentos.

Com relação à abordagem da pirâmide da atividade física, foi observado, inicialmente, que os escolares não tinham conhecimento prévio sobre 
esse instrumento metodológico. Desenvolvido recentemente por Martins (2009), o conceito de adaptação da pirâmide da atividade física para crianças e adolescentes faz uma abordagem categorizada e representativa de diversos grupos de atividades, por exemplo, as atividades físicas diárias, na base da pirâmide, que devem compreender a maior parte do tempo diário das crianças. No segundo nível, os exercícios aeróbicos, na forma de algum esporte, que também devem ser executados com regularidade, enquanto os exercícios que envolvem o fortalecimento ósseo e muscular, representados no terceiro nível, devem ser incorporados às atividades semanais. Por fim, compondo o ápice da pirâmide (menor área), estão as atividades sedentárias que devem ser evitadas sempre que possível. Por associação ao conhecimento prévio adquirido na análise da pirâmide alimentar, os escolares demonstraram rápida compreensão das informaçôes teóricas contidas na pirâmide de atividade física. Houve rápida percepção da importância da prática diária, da diversificação e frequência dos exercícios físicos necessários ao desenvolvimento adequado de crianças e adolescentes. Neste contexto, foi enfatizada a relevância da inclusão de exercícios aeróbicos e de fortalecimento ósseo e muscular na rotina de atividades, e dos malefícios decorrentes de atividades sedentárias por longo período de tempo.

As atividades físicas desenvolvidas na forma de jogos e brincadeiras mostraram ser um instrumento metodológico eficaz na disseminaçáo do conhecimento relacionado à qualidade de vida, uma vez que foi capaz de prender a atenção dos alunos durante as explicaçóes e discussões, além de estimular a reflexão de seus hábitos de atividade física ao participarem, com empolgação e interesse, dos jogos e brincadeiras propostos. O estímulo constante da realização de exercícios físicos entre crianças e adolescentes deve ser um processo contínuo de combate ao sedentarismo, pois reforça a necessidade de adotar medidas de prevenção que contribuam para a diminuição da ocorrência de doenças relacionadas à inatividade física, cujo desenvolvimento iniciado na infância ou adolescência pode se manifestar gravemente na fase adulta (HALLAH et al., 2006).

Cabe aos profissionais de saúde a identificação dos casos de subnutrição e de excesso de peso e o seu devido tratamento. Contudo, as açóes dos professores, e da própria escola, como agentes ativos em um processo que integra saúde-ensino, por meio da educação nutricional 
e o incentivo à prática de atividade física, são fundamentais para o sucesso dos programas de incentivo à melhoria da qualidade de vida. A orientação por parte dos professores sobre estilo de vida saudável pode contribuir para aumentar o consumo de frutas e verduras bem como a realização de atividades físicas. Neste sentido, o presente estudo foi capaz de fornecer aos alunos conteúdos sobre alimentação saudável e de atividade física, de forma lúdica e integrativa, tornando-os agentes ativos no processo de ensino-aprendizagem ao invés de expectadores apenas assimilando informaçôes.

Em resumo, os resultados obtidos neste estudo evidenciaram não somente a elevada prevalência de excesso de peso entre os escolares na faixa de 10 a 13 anos, do $5^{\circ}$ ano do ensino fundamental, mas também o baixo peso e a baixa estatura para a idade em algumas crianças. De modo geral, foi constatado que a alimentação incluiu o consumo frequente de elementos essenciais, como arroz, feijáo, carnes e frutas, porém houve baixo consumo de verduras, e consumo regular de refrigerantes e biscoitos. Embora tenha havido discernimento de quais alimentos não faziam bem à saúde, foi enfatizado, junto aos escolares, a importância de não apenas ter o conhecimento sobre os hábitos saudáveis, mas da necessidade de aplicá-los no dia-dia. Foi evidenciado ainda que os alunos exercem prática regular de atividade física, principalmente fora do ambiente escolar e sem orientação profissional, entretanto, também possuem hábitos sedentários ligados ao chamado "tempo de tela", ou seja, ficar por mais de duas horas em frente à televisão ou computadores.

A realização das atividades e discussóes propostas criou uma excelente oportunidade de reforço do conhecimento dos escolares sobre os benefícios de uma alimentação saudável e equilibrada, além de demonstrar que a prática de exercícios físicos pode ser divertida e prazerosa, tornando-se uma forma simples e prática de manter uma vida saudável e prevenir o aparecimento de doenças em outros estágios da vida.

\section{AGRADECIMENTOS}

À Pró-Reitoria de Assuntos Comunitários da Universidade Federal da Paraíba pelo apoio. 


\section{REFERÊNCIAS}

ALVARENGA, W. D. A. et al. Fatores determinantes e condicionantes para o sobrepeso e a obesidade em pré-escolares: uma revisão integrativa. Revista Interdisciplinar, Teresina, v. 6, n. 4, p. 216222, out.-dez.2014. Disponível em: <https://revistainterdisciplinar. uninovafapi.edu.br/index.php/revinter/article/viewFile/228/pdf_86>. Acesso em: 10 maio 2017.

ALVES, U. S. Não ao sedentarismo, sim à saúde: contribuiçóes da educação física escolar e dos esportes. O Mundo da Saúde, São Paulo, v. 31, n. 4, p. 464-469, out.-dez. 2007. Disponível em: <https://www. saocamilo-sp.br/pdf/mundo_saude/56/01_nao_ao_sedentarismo. pdf>. Acesso em: 10 abr. 2017.

AMIGO, H. et al. Factores condicionantes de la estatura en escolares de alta vulnerabilidad social. Impresos Maigret. Santiago: Maigret, 1994.

ANDERSEN, R. E. et al. Relationship of physical activity and television watching with body weight and level of fatness among children: results from the Third National Health and Nutrition Examination Survey. JAMA, v. 279, n. 12, p. 938-942, mar. 1998. doi: 10.1001/jama.279.12.938.

ARAÚJO, E. D. D. S. Estado nutricional e adiposidade de escolares de 7 a 14 anos das cidades de Florianópolis-SC e PelotasRS. Brazilian Journal of Kinanthropometry and Human Performance, Florianópolis, v. 3, n. 1, p. 111, 2001. doi: http:// dx.doi.org/10.5007/\%25x.

ASSIS, M. A. et al. Validation of the third version of the Previous Day Food Questionnaire (PDFQ-3) for 6-to-11-years-old schoolchildren. Cadernos de Saúde Pública, Rio de Janeiro, v. 25, n. 8, p. 1816-1826, ago. 2009. doi: http://dx.doi.org/10.1590/S0102311X2009000800018.

BARQUERA, S. et al. Coexistence of maternal central adiposity and child stunting in Mexico. International Journal of Obesity, Boston, v. 31, n. 4, p. 601-607, 2007. doi: 10.1038/sj.ijo.0803529.

BRASIL. Ministério da Saúde. Secretaria de Atenção à Saúde. 
Departamento de Atenção Básica. Guia alimentar para a população brasileira. 2. ed., Brasília, DF: Ministério da Saúde, 2014. 156 p.

CDCP - Center for Diseases Control and Prevention. School health guidelines to promote healthy eating and physical activity. MMWR, Atlanta, v. 16. n. 5, p. 1-77, 2011.

COELHO, L. G. et al. Association between nutritional status, food habits and physical activity level in schoolchildren. Jornal de Pediatria, Porto Alegre, v. 88, n. 5, p. 406-12, set.-out. 2012. doi: http://dx.doi.org/10.2223/JPED.2211.

ENES, C. C.; SLATER, B. Obesity in adolescence and its main determinants. Revista Brasileira de Epidemiologia, São Paulo, v. 13, n. 1, p. 163-71, mar. 2010. doi: http://dx.doi.org/10.1590/ S1415-790X2010000100015.

FERNALD, L. C.; NEUFELD, L. M. Overweight with concurrent stunting in very young children from rural Mexico: prevalence and associated factors. European Journal of Clinical Nutrition, v. 61, n. 5, p. 623-632, May 2007. doi: 10.1038/sj.ejcn.1602558.

FISBERG, R. M. et al. Índice de qualidade da dieta: avaliação da adaptação e aplicabilidade. Revista de Nutrição, Campinas, v. 17. n. 3, p. 301-318, jul. 2004. doi: http://dx.doi.org/10.1590/S141552732004000300003 .

GABRIEL, C. G.; SANTOS, M. V. D.; VASCONCELOS, F. D. A. G. D. Avaliação de um programa para promoção de hábitos alimentares saudáveis em escolares de Florianópolis, Santa Catarina, Brasil. Revista Brasileira de Saúde Materno Infantil, Recife, v. 8, n. 3, p. 299-308, 2008. doi: http://dx.doi.org/10.1590/S151938292008000300009 .

GOLDBERG, J. P. et al. The obesity crisis: don't blame it on the pyramid. Journal of the American Dietetic Association, v. 104, n. 7, p. 1141-1147, Jul. 2004. doi: 10.1016/j.jada.2004.04.026.

GUTHOLD, R. et al. Physical activity and sedentary behavior among schoolchildren: a 34-country comparison. The Journal of Pediatrics, v. 157, n. 1, p. 43-49 e1, Jul. 2010. doi: 10.1016/j.jpeds.2010.01.019.

$\mathrm{HA}$, A. et al. Eating and physical activity practices in risk of overweight 
and overweight children: compliance with US Department of Agriculture food guide pyramid and with National Association for Sport and Physical Activity guidelines for children. Nutrition Research, v. 25, n. 10, p. 905-915, 2005. doi: http://dx.doi. org/10.1016/j.nutres.2005.09.010.

HALLAL, P. C. et al. Prevalence of sedentary lifestyle and associated factors in adolescents 10 to 12 years of age. Cadernos de Saúde Pública, Rio de Janeiro, v. 22, n. 6, p. 1277-1287, jun. 2006. doi: http://dx.doi.org/10.1590/S0102-311X2006000600017.

HUNT, J. B. Childhood obesity and academic outcomes. Institute for Educational Leadership and Policy. 2008. Disponível em: <http:// www.huntinstitute. org/elements/media/files/Hunt_Obesity_Memo. pdf>. Acesso em: 4 mar. 2017.

IBGE - Instituto Brasileiro de Geografia e Estatística. Pesquisa de Orçamentos Familiares 2008-2009: Antropometria e Estado Nutricional de Crianças, Adolescentes e Adultos no Brasil. Rio de Janeiro, 2010.

JARDIM, J. B.; DE SOUZA, I. L. Obesidade infantil no Brasil: uma revisão integrativa. Journal of Management \& Primary Health Care, Recife, v. 8, n. 1, p. 66-90, 2017. Disponível em: <http://www. jmphc.com.br/saude-publica/index.php/jmphc/article/view/275>. Acesso em: 15 mar. 2017.

KAIN, B. et al. Validación y aplicación de instrumentos para evaluar intervenciones educativas en obesidad de escolares. Revista Chilena de Pediatría, Santiago, v. 72, n. 4, p. 308-318, jul. 2001. doi: http:// dx.doi.org/10.4067/S0370-41062001000400005.

KENNEDY, E. T. et al. Popular diets: correlation to health, nutrition, and obesity. Journal of the American Dietetic Association, v. 101, n. 4, p. 411-20, Apr 2001. doi: 10.1016/S0002-8223(01)00108-0.

LAMAS, R.; LORENZO, T. Prevenção da obesidade em crianças e adolescentes em Cuba. In: FISBERG, M. (Org.). Atualizaçáo em obesidade na infância e adolescência. São Paulo: Atheneu, 2004. $218 \mathrm{p}$.

LEE, Y. S. Consequences of childhood obesity. Annals 
Academy of Medicine Singapore, v. 38, n. 1, p. 75-77, Apr. 2009. Disponível em: <https://pdfs.semanticscholar. org/51b9/11cf27bdd03fbb817e8baac86e19260aa72c.pdf>. Acesso em: 15 maio 2017.

LICHTENSTEIN, A. H. et al. Diet and lifestyle recommendations revision 2006: a scientific statement from the American Heart Association Nutrition Committee. Circulation, Waltham, v. 114, n. 1, p. 82-96, Jul. 2006. doi: 10.1161/CIRCULATIONAHA.106.176158.

LOHMAN, T. G. Skinfolds and body density and their relation to body fatness: a review. Human Biology, Detroit, v. 53, n. 2, p. 181225, May 1981.

MALTA, D. C. et al. Prevalência de fatores de risco e proteção de doenças crônicas não transmissíveis em adolescentes: resultados da Pesquisa Nacional de Saúde do Escolar (PeNSE), Brasil, 2009. Ciência \& Saúde Coletiva, Rio de Janeiro, v. 15, n. 2, p. 3009-3019, out. 2010. doi: http://dx.doi.org/10.1590/S1413-81232010000800002.

MALTA, D. C.; MORAIS NETO, O. L. D.; SILVA JUNIOR, J. B. D. Apresentação do Plano de açôes estratégicas para o enfrentamento das doenças crônicas não transmissíveis no Brasil, 2011 a 2022. Epidemiologia e Serviços de Saúde, Brasília, v. 20, n. 4, p. 425438, 2011.

MARTINS, C. O. Pirâmide da atividade física para crianças e adolescentes. Laboratório de Estudos e Pesquisas em Atividade Física e Saúde, João Pessoa-PB, 2009. Disponível em: $<$ http://carolinemartins.yolasite.com/resources/aula2.pdf>. Acesso em: Acesso em 30/08/2016.

MARTINS, V. J. et al. Long-lasting effects of undernutrition. International Journal of Environmental Research and Public Health, Boston, v. 8, n. 6, p. 1817-1846, 2011. doi: 10.1038/ sj.ijo.0803529.

MAYER, A. P. F.; WEBER, L. D. A influência da escola na alimentação e na atividade física da criança. CAMINE: Caminhos da Educação, Franca, v. 5, n. 1, p 1-19, 2013. Disponível em: <https://ojs.franca. unesp.br/index.php/caminhos/article/view/682>. Acesso em: 10 abr. 2017. 
MCDONALD, C. M. et al. The effect of multiple anthropometric deficits on child mortality: meta-analysis of individual data in 10 prospective studies from developing countries. The American Journal of Clinical Nutrition, Rockville, v. 97, n. 4, p. 896-901, Apr. 2013. doi: 10.3945/ajen.112.047639.

MELLO, E. D.; LUFT, V. C.; MEYER, F. Childhood obesity-towards effectiveness. Jornal de Pediatria, Porto Alegre, v. 80, n. 3, p. 173182, maio-jun. 2004. doi: http://dx.doi.org/10.2223/JPED.1180.

MENDONCA, C. P.; DOS ANJOS, L. A. Dietary and physical activity factors as determinants of the increase in overweight/obesity in Brazil. Cadernos de Saúde Pública, Rio de Janeiro, v. 20, n. 3, p. 698-709, maio-jun. 2004.

MUST, A.; TYBOR, D. J. Physical activity and sedentary behavior: a review of longitudinal studies of weight and adiposity in youth. International Journal of Obesity, London, v. 29 Suppl 2, p. S8496, Sep. 2005.

OGDEN, C. L. et al. Prevalence of childhood and adult obesity in the United States, 2011-2012. JAMA, v. 311, n. 8, p. 806-814, 2014. doi: 10.1001/jama.2014.732.

ONIS, M. WHO Child Growth Standards based on length/height, weight and age. Acta Paediatr Suppl., v. 95, n. S450, p. 76-85, Apr. 2006. doi: 10.1080/08035320500495548.

ONIS, M. D. et al. Development of a WHO growth reference for school-aged children and adolescents. Bulletin of the World Health Organization, Bethesda, v. 85, n. 9, p. 660-667, 2007. doi: 10.2471/ BLT.07.043497.

RIVERA, J. A. et al. Childhood and adolescent overweight and obesity in Latin America: a systematic review. Lancet Diabetes Endocrinol, v. 2, n. 4, p. 321-332, Apr. 2014. doi: 10.1016/S22138587(13)70173-6.

SAWAYA, A. L. et al. Os dois Brasis: quem são, onde estão e como vivem os pobres brasileiros. Estudos avançados, São Paulo, v. 17, n. 48, p. 21-44, maio-ago. 2003. doi: http://dx.doi.org/10.1590/ S0103-40142003000200003. 
SOUSA, C. P.; OLINDA, R. A.; PEDRAZA, D. F. Prevalence of stunting and overweight/obesity among Brazilian children according to different epidemiological scenarios: systematic review and metaanalysis. Sáo Paulo Medical Journal, São Paulo, v. 134, n. 3, p. 251-262, maio-jun. 2016. doi: http://dx.doi.org/10.1590/15163180.2015 .0227121 .

TAVELLI, S. et al. Sources of error and nutritional adequacy of the food guide pyramid. Journal of American College Health, Carbondale, v. 47, n. 2, p. 77-82, Sep. 1998. Doi: https://doi. org/10.1080/07448489809595623

TRICHES, R. M.; GIUGLIANI, E. R. J. Obesidade, práticas alimentares e conhecimentos de nutrição em escolares. Revista de Saúde Pública, São Paulo, v. 39, n. 4, p. 541-547, 2005. doi: http:// dx.doi.org/10.1590/S0034-89102005000400004.

VELASQUEZ-MELENDEZ, G. et al. Mild but not light or severe food insecurity is associated with obesity among Brazilian women. The Journal of Nutrition, Rockville, v. 141, n. 5, p. 898-902, May 2011.

VEUGELERS, P. J.; FITZGERALD, A. L. Effectiveness of school programs in preventing childhood obesity: a multilevel comparison. American Journal of Public Health, Rockville, v. 95, n. 3, p. 432-5, Mar. 2005. doi: 10.2105/AJPH.2004.045898.

WHO - World Health Organization. Physical status: the use of and interpretation of anthropometry. 1995. Disponível em: <http://www. who.int/childgrowth/publications/physical_status/en/>. Acesso em: 16 maio. 2017.

Global strategy on diet, physical activity and health. 2004. Disponível em: <http://www.who.int/dietphysicalactivity/strategy/ eb11344/strategy_english_web.pdf>. Acesso em: 16 maio 2017.

. WHO child growth standards: length/height for age, weight-for-age, weight-for-length, weight-for-height and body mass index-for-age, methods and development. 2006. Disponível em: < http://www.who.int/childgrowth/standards/technical_report/en/>. Acesso em: 20 maio 2017. 
- Global status report of non-communicable diseases 2014.

2014. Disponível em: <http://www.who.int/nmh/publications/ncdstatus-report-2014/en/>. Acesso: 18 mar. 2017.

Obesity and Overweight. 2016. Disponível em: <http:// www.who.int/mediacentre/factsheets/fs311/en/>. Acesso em: 30 ago. 2016.

WILLETT, W. C.; STAMPFER, M. J. Rebuilding the food pyramid. Scientific American, v. 288, n. 1, p. 64-71, Jan. 2003. doi: 10.1038/ scientificamerican1206-12sp.

ZHANG, G. et al. Television watching and risk of childhood obesity: a meta-analysis. European Journal of Public Health, v. 26, n. 1, p. 13-18, Feb. 2016. doi: 10.1093/eurpub/ckv213.

Submetido em 2 de junho de 2017.

Aprovado em 13 de outubro de 2017. 